\title{
Space User Visibility Benefits of the Multi-GNSS Space Service Volume: An Internationally- Coordinated, Global and Mission-Specific Analysis
}

\author{
Werner Enderle, ESOC, European Space Agency \\ Francesco Gini, ESOC, European Space Agency \\ Henno Boomkamp, ESOC, European Space Agency \\ Joel J. K. Parker, Goddard Space Flight Center, NASA \\ Benjamin W. Ashman, Goddard Space Flight Center, NASA \\ Bryan W. Welch, Glenn Research Center, NASA \\ Mick Koch, Glenn Research Center, NASA \\ O. Scott Sands, Glenn Research Center, NASA
}

\section{BIOGRAPHIES}

Werner Enderle is the Head of the Navigation Support Office at ESA's European Space Operations Center (ESOC) in Darmstadt, Germany. Previously, he worked at the European GNSS Authority (GSA) as the Head of System Evolutions for Galileo and EGNOS and he also worked for the European Commission in the Galileo Unit. Since more than 20 years, he is involved in activities related to the use of GPS/GNSS for space applications. He holds a doctoral degree in aerospace engineering from the Technical University of Berlin, Germany.

Francesco Gini is a Navigation Engineer at the Navigation Support Office (OPS-GN) at the European Space Operations Centre (ESOC) of ESA. He is responsible for the Space Service Volume (SSV) and Precise Orbit Determination (POD) related activities. He received his $\mathrm{PhD}$ in Astronautics and Satellite Sciences at the University of Padova in 2014 and since then he has been working in ESOC.

Henno Boomkamp has worked on precise orbit determination and satellite tracking data analysis for more than two decades. He chaired the Low Earth Orbiter Working Group of the International GNSS Service for seven years, as well as a Working Group on precise orbit determination of the International Association of Geodesy (IAG).

Joel Parker is the Positioning, Navigation, and Timing (PNT) Policy lead at NASA Goddard Space Flight Center, where he develops and promotes civil space use of GNSS on behalf of NASA Space Communications and Navigation (SCaN). He has worked as an Aerospace Engineer at NASA since 2010.

Benjamin W. Ashman is an Aerospace Engineer at the NASA Goddard Space Flight Center, where he supports numerous space communication and navigation efforts, most recently as part of the OSIRIS-REx navigation team. His research has primarily been focused on space applications of GPS since starting at the agency in 2015.

Bryan W. Welch is the Technical Lead for communication systems modelling and simulation at NASA Glenn Research Center, where he leads the analysis development, verification, and validation activities of the SCaN Center for Engineering, Networks, Integration, and Communications Project. He has worked as an Electronics Engineer at NASA since 2002.

Mick Koch is an Electronics Engineer at the NASA Glenn Research Center, where he supports Positioning, Navigation, and Timing efforts and other Space Communications and Navigation projects. Recent research includes experiments with GPS reflectometry and beam pattern extrapolation. He has worked at NASA since 2016.

O. Scott Sands has been a Communications System Analyst at NASA, Glenn Research Center since 2000 where he develops space navigation, sensing and communications systems. Scott currently leads Position Navigation and Timing Policy related activities at GRC in support of the NASA's Space Communications and Navigation Office. 


\begin{abstract}
The number and scope of Global Navigation Satellite System (GNSS)-based space applications has grown significantly since the first GNSS space receiver was flown in the early 1980's. The vast majority of GNSS space users operate in Low-Earth Orbit (LEO), where the use of GNSS receivers has become routine. However, the use of GNSS has expanded to other orbit regimes like Geostationary Orbits (GEO) and High Eccentric Orbits (HEO) but has been very limited due to the challenges involved. The major challenges for such types of orbits including much weaker signals, reduced geometric diversity, and limited signal availability. In any case, considering the recent development of multiple GNSS constellations and ongoing upgrades to existing constellations, GNSS signal availability will improve significantly. As a result, this expanded multi-GNSS signal capability will enable improved on-orbit navigation performance and will also allow the development of new mission concepts. High altitude space users will especially benefit from this evolution, which will provide GNSS signals to challenging regimes well beyond Low Earth Orbit. These benefits will only be realised, however, if additional signals are designed to be interoperable, are clearly documented and supported.
\end{abstract}

In order to enhance the overall GNSS performance for spacecraft's in regimes from LEO, GEO to HEO and beyond, all Satellite Navigation constellation providers and regional augmentation system providers are working together through the United Nations International Committee on GNSS (ICG) forum to establish an interoperable GNSS Space Service Volume (SSV) for the benefit of all GNSS space users.

This paper provides an overview of the technical work and in particular the simulations, performance analysis and discussions of the outcomes and results obtained by the UN ICG Working Group-B in the context of the GNSS Space Service Volume activities, which were supported by all GNSS service providers.

\title{
INTRODUCTION
}

Global navigation satellite systems (GNSS), which were originally designed to provide positioning, velocity, and timing services for terrestrial users, are now also being increasingly utilized for autonomous navigation in space. Historically, most space users have been located at low altitudes, where GNSS signal reception is similar to that on the ground. More recently, however, users are relying on these signals at high altitudes, near to or above the GNSS constellations themselves; in the so-called Space Service Volume (SSV).

Space receivers in the GNSS SSV operate in an environment significantly different than the environment of a classical terrestrial receiver or GNSS receiver in Low Earth Orbit. SSV users span very dynamic and changing environments when traversing above and below the altitudes of the GNSS constellations. Users located in orbits with an altitude below the altitude of the GNSS constellations can make use of direct line of sight (LoS) signals, while those above the orbit of the GNSS constellations must rely on GNSS signals transmitted from the other side of the Earth, passing over the Earth's limb. These "above the constellation" space users experience higher user ranging error, reduced user-received power levels, significantly reduced satellite visibility, less optimal geometric diversity, and in case of elliptical orbits, highly-dynamic motion in terms of difference of the velocity for the apogee and the perigee. In these environments, an increased number of available GNSS signals of sufficient power and accuracy would substantially improve the potential signal visibility, and thus mission navigation performance.

The United Nations' sponsored International Committee on GNSS (ICG) has been working diligently for nearly a decade and a half to ensure that GNSS signals and systems are interoperable. A detailed overview of policy aspects related to this subject topic is provided in the paper from Parker et. al. [2], whereas this paper is concentrating on the technical aspects of the interoperable GNSS SSV. The ICG defines interoperability as "the ability of global and regional navigation satellite systems, and augmentations and the services they provide, to be used together to provide better capabilities at the user level than would be achieved by relying solely on the open signals of one system". One initiative being taken by the ICG is to ensure that GNSS signals within the SSV are available and interoperable across all international global constellations and regional augmentations. This initiative is being carried out within the ICG Working Group-B (WG-B) on "Enhancement of GNSS Performance, New Services and Capabilities". Signal characteristics supporting the SSV for each of these GNSS constellations and regional augmentations are detailed in Table 1 and in the UN SSV booklet [1].

The benefits of employing an interoperable, multi-constellation GNSS capability at these higher altitudes are numerous, including more precise, real-time position, velocity, and timing knowledge on-orbit; increased resiliency due to multi-GNSS signal diversity; reduced reliance on ground support infrastructure; increased responsiveness to trajectory manoeuvres and enhanced vehicle autonomy resulting in improved on-orbit agility; and the ability to utilize lower-cost components such as onboard clocks. 
Spacecraft expansion into the SSV has spawned exciting new operational missions through radically improved navigation and timing performance, quick trajectory manoeuvre recovery, and improved space vehicle autonomy. But currently these missions have all employed a single GNSS constellation. Expansion by employing multi-GNSS interoperable signals will be transformative for missions of the future.

This paper reports on a series of simulations and associated analyses that were performed in a cooperative effort among all GNSS operators, via their participation in the ICG WG-B. The key objectives of these simulations were to demonstrate and quantify the improvements in signal availability obtained by multi-constellation GNSS receivers within the SSV, in comparison to single constellation receivers, and to establish a collectively agreed framework for doing such simulations among all participating agencies.

For this reason, the development of consistent simulation capabilities was of fundamental importance. In order to achieve this objective, a stepwise approach (starting simple and moving to complex) for the development of the tools used within the simulations was agreed. The applied simulation approach took into account the need for statistical data on a global scale (grid), but with simple assumptions for the scenarios. This was realized by simulations and analysis within the Global SSV. On the other side, it was also important to cover realistic mission scenarios, taking into account more complex assumptions and calculations. This aspect was covered by the selected three mission-specific scenarios for GEO, HEO and Lunar. The focus of the simulations was on the important SSV parameters Signal Availability and Maximum Outage Duration.

\section{DEFINITION OF THE INTEROPERABLE GNSS SPACE SERVICE VOLUME}

Historically, most space users have been located at low altitudes, where Global Navigation Satellite System (GNSS) signal reception is similar to that on the ground. More recently, however, users are relying on these signals at high altitudes, near to or above the GNSS constellations themselves. The availability and performance of GNSS signals at high altitude is documented as the GNSS Space Service Volume (SSV). While different definitions of the SSV exists and may continue to exist for the different service providers, within the context of the UN SSV booklet the SSV is defined as the region of space between 3,000 km and $36,000 \mathrm{~km}$ above the Earth's surface, which is from low Earth orbit $(3,000 \mathrm{~km})$ to geostationary altitude $(36,000 \mathrm{~km})$. The relevant regions of the GNSS SSV are depicted in Figure 1, along with the altitude ranges of the contributing GNSS constellations that are located in Medium-Earth Orbit (MEO). It is noted that some GNSS constellations and regional augmentations also offer satellites at Geostationary Orbits (GEO) and/or Inclined Geosynchronous Orbits (IGSO). As shown, the SSV encompasses a large range of altitudes, above and below the GNSS constellations and regional augmentations. In order to accommodate the differing needs of SSV users, the SSV is subdivided into two regions that have different characteristics in terms of the geometry and quantity of signals available to users in those regions:

1. Lower Space Service Volume for Medium Earth Orbits: 3,000-8,000 km altitude. GNSS space receivers located between 3,000 km and 8,000 km altitude can receive GNSS signals from the spacecraft nadir direction and the spacecraft zenith direction with respect to the Earth. Zenith signals are received in-line with Low-Earth Orbit (LEO) spacecraft and Earth-based GNSS signal reception. The signals arriving from spacecraft nadir are emitted by GNSS satellites located at the opposite side of the Earth and pass the limb of the Earth before arriving at the receiver.

2. Upper Space Service Volume for Geostationary and High Earth Orbits: 8,000-36,000 km altitude. The upper SSV primarily accommodates users in Geostationary and High Earth Orbits. It is characterized by significantly reduced signal received power and therefore signal availability. This is primarily because most signals are traveling across the limb of the Earth. Space Users that employ advanced technology, like more robust antennas and receivers with weak signal processing capabilities can also receive and process GNSS signals in altitudes higher than $36,000 \mathrm{~km}$.

Within these regions, the performance of a single GNSS constellation or combination of constellations for a particular mission is determined by three parameters: Pseudorange accuracy, Received signal power, and Signal availability for 1 signal and 4 signals simultaneously. These three parameters are interrelated; if a signal is too weak, if Earth blocks the signal, or if the signal 
does not have sufficient accuracy, it is not considered as available. The geometry for signal reception in the lower SSV and also

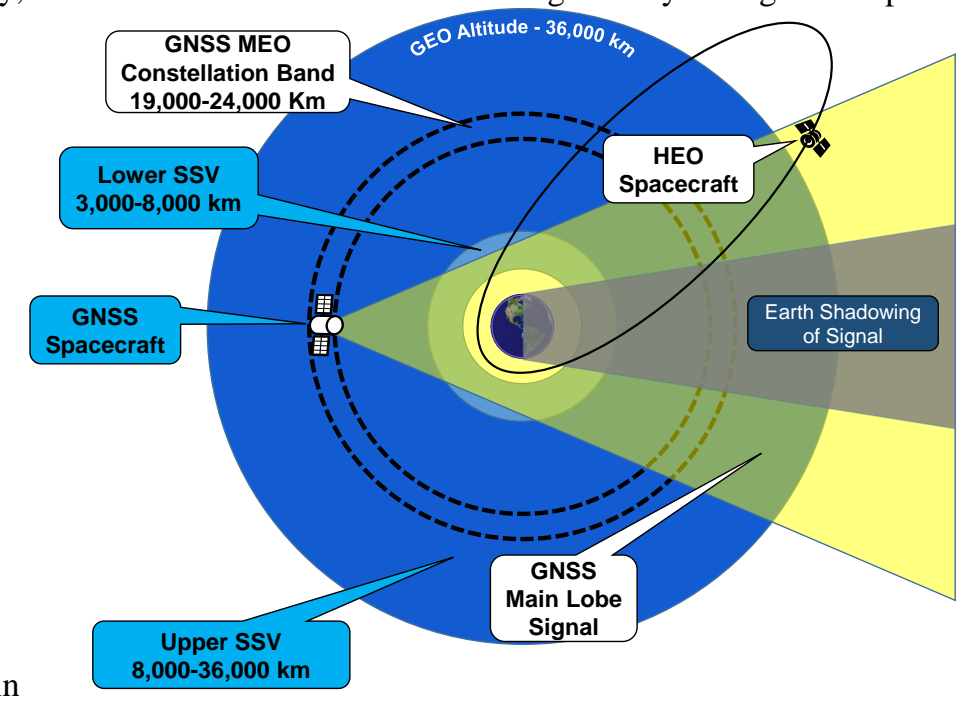

the upper SSV region is shown in

Figure 1.

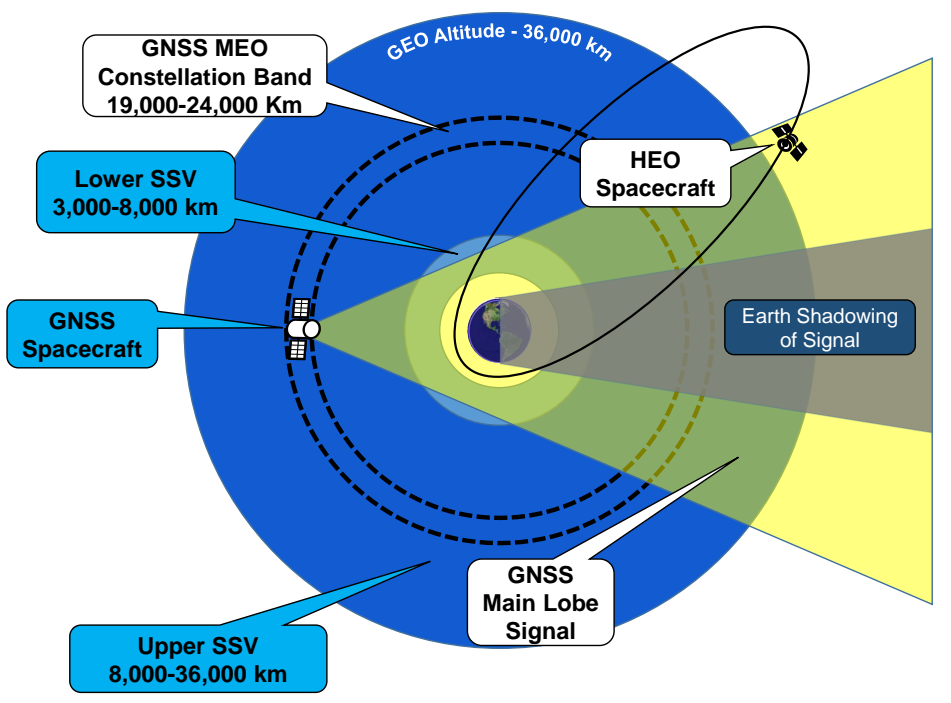

Figure 1: The GNSS Space Service Volume and its regions

\section{SIMULATIONS CONCEPT AND CONDUCTED ANALYSIS}

Satellite navigation constellation providers and regional augmentation system providers are working together via the United Nations International Committee on GNSS (ICG) to establish an interoperable multi-GNSS Space Service Volume (SSV) for the benefit of all GNSS space users. One key facet of this effort is an internationally-coordinated simulation of GNSS singleand multiple-constellation performance expectations in the Space Service Volume (SSV), based on the individual constellation signal characteristics. Simulation ground rules and assumptions were developed and agreed upon by the ICG team. Within the ICG, each national team performed global and mission-specific analyses independently, and the results were compared to provide a measure of independent verification. Ultimately, this approach enabled each national team to develop their own SSV simulation capability across the multi-GNSS constellations which can now be used as a foundation for future internationally-coordinated analyses. The key objectives of these simulations were to demonstrate and quantify the improvements in signal availability obtained by using multi-constellation GNSS receivers within the SSV. 


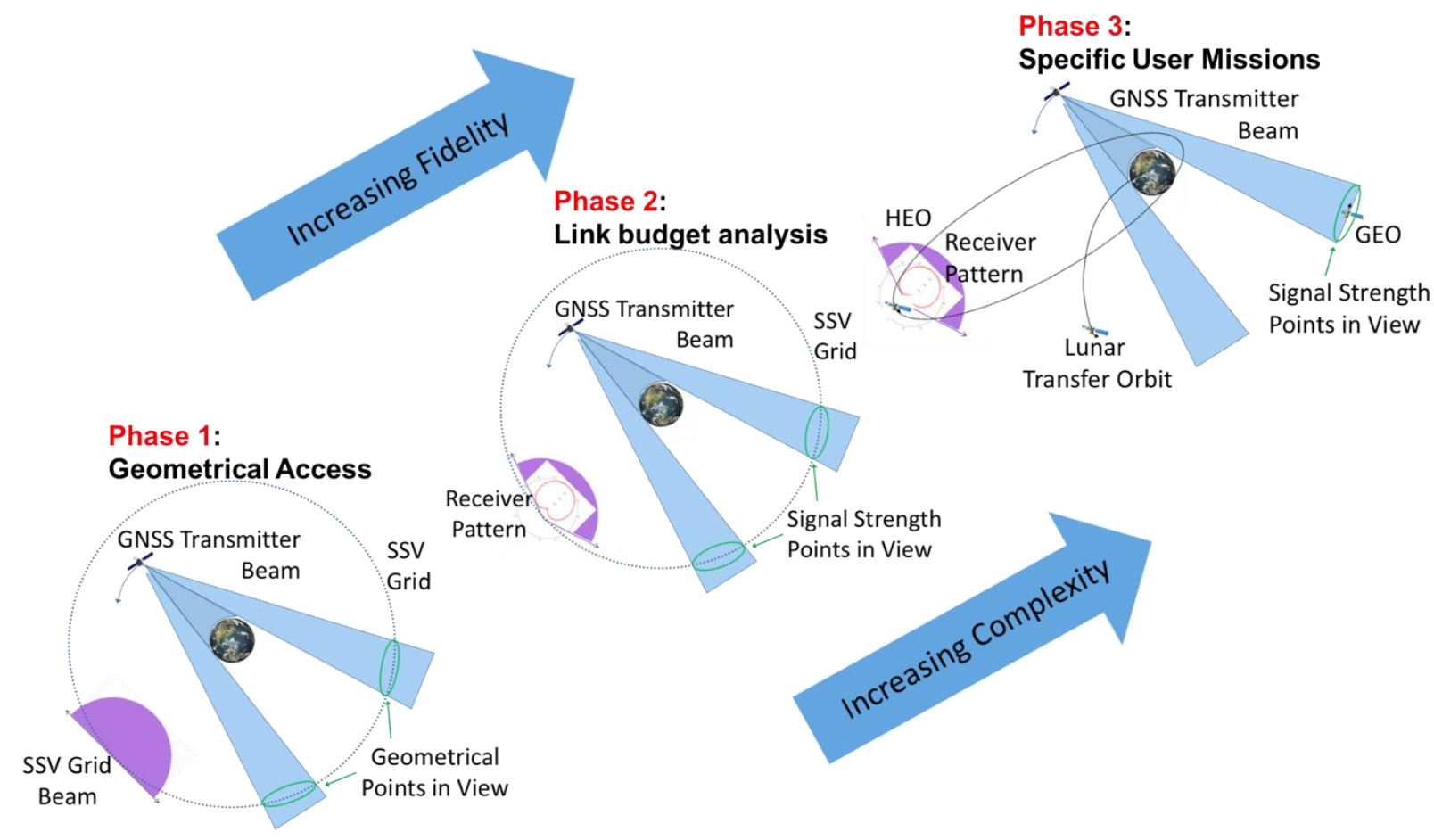

Figure 2: Phased approach for Simulations

The general approach for the simulations was structured in two general cases and three phases. The three phases are outlined in Figure 2. The basic principle for these three phases was to start with the simplest concept, namely to consider for Phase 1 only the relative-geometry between the space user and the GNSS constellations. For Phase 2, the link budget and related general assumptions about the GNSS receiver characteristics (especially the signal to noise ratio C/N0 threshold for signal acquisition) was also considered. Phase 3 was intended to be the most realistic case and therefore three representative missions were defined. Also, the link budget, space user 3-D attitude, the locations of the GNSS antennas on the space user spacecraft, the pointing directions of the antennas, and the performance characteristics of representative commercial off-the-shelf GNSS receiver and antenna products were used for the simulations. Two general analyses were performed: a global SSV performance case for the Upper and Lower SSV, and a mission-specific SSV performance case consisting of three scenarios. Global performance was estimated by simulating signal availability at a fixed grid of points in space (see Figure 6), at both the lower SSV altitude of $8,000 \mathrm{~km}$, and the upper SSV at $36,000 \mathrm{~km}$. The availability was then calculated by simulating navigation receiver operations over a two-week period, and over all the points in each grid. This can be interpreted as a measure of the performance that space missions can expect while employing GNSS in the SSV. Mission specific performance estimates were obtained by estimating signal availability for a spacecraft on a particular trajectory within the SSV. Mission-specific scenarios considered in this study included: 1) geostationary orbit, 2) a highly-elliptic orbit, and 3) a lunar trajectory. The purpose of this phase of analysis was to provide "real-world" estimates for a concrete mission using similar methods to those used for estimation of global performance. In total, this information will provide prospective SSV users simulation results that demonstrate the benefits and possibilities offered by an inter-operable SSV.

\section{Assumptions for GNSS Constellations Configuration}

To ensure a consistent set of capabilities across all Global Navigation Satellite System (GNSS) constellations, a Space Service Volume (SSV) capabilities template has been completed by each GNSS service provider to capture their contributions to each of the significant parameters used in the analyses. On-orbit flight results will differ from these characteristics due to missionspecific geometry, receiver sensitivity, time-dependent service characteristics, and other factors. In all cases, only services provided by the main-lobe signal was captured here. Table 1 presents an overview of the configuration of each constellation, including operational status, constellation configuration, and general orbit parameters. In addition to this information, each GNSS Service Provider provided SSV signal characteristics for their system, in terms of signal band and frequency, minimum received civilian signal power at a GEO user location, and maximum off-boresight angle (maximum beam-width) of the main lobe. These values are summarized in Table 2. As shown in this table, the GNSS satellites occupy different orbits, in particular: Medium Earth Orbit (MEO), Geosynchronous Equatorial Orbit (GEO), Inclined Geosynchronous Orbit (IGSO). In case of Galileo, the FOC satellites are the Full Operational Capability satellites. 


\begin{tabular}{|c|c|c|c|c|c|c|c|}
\hline $\begin{array}{c}\text { System } \\
\text { name }\end{array}$ & Nation & Coverage & Status & $\begin{array}{l}\text { No. freq. } \\
\text { and } \\
\text { signals }\end{array}$ & $\begin{array}{c}\text { No. spacecraft } \\
\text { Nominal / } \\
\text { orbital planes }\end{array}$ & $\begin{array}{l}\text { Semi-major } \\
\text { axis }(\mathbf{k m})\end{array}$ & $\begin{array}{c}\text { Inclin. } \\
\left({ }^{\circ}\right)\end{array}$ \\
\hline GPS & USA & Global & Operational & $3 / 4$ & $24 / 6$ & 26560 & 55 \\
\hline GLONASS & Russia & Global & Operational & $2 / 6$ & $24 / 3$ & 25510 & 64.8 \\
\hline Galileo & $\begin{array}{l}\text { European } \\
\text { Union }\end{array}$ & Global & $\begin{array}{c}\text { Operational } \\
\text { Initial Service: } \\
2016 \\
\text { FOC planned: } \\
2020\end{array}$ & $5 / 10$ & $24 / 3$ & 29600 & 56 \\
\hline BDS & China & Global & $\begin{array}{c}\text { Operational: } \\
\text { Regional FOC } \\
2012 \\
\text { In build-up: } \\
\text { Global 2018-2020 }\end{array}$ & $3 / 5$ & $\begin{array}{l}\text { MEO: } 24 / 3 \\
\text { IGSO: } 3 / 3 \\
\text { GEO: } 5 / 1\end{array}$ & $\begin{array}{l}27906 \\
42164 \\
42164\end{array}$ & $\begin{array}{c}55 \\
55 \\
0\end{array}$ \\
\hline QZSS & Japan & $\begin{array}{c}\text { Regional } \\
\text { (Japan) }\end{array}$ & In build-up: 2018 & $4 / 7$ & $\begin{array}{l}\text { HEO: } 3 / 3 \\
\text { GEO: } 1 / 1\end{array}$ & 42164 & $\begin{array}{c}40 \\
0\end{array}$ \\
\hline NaviC & India & $\begin{array}{c}\text { Regional } \\
\text { (India) }\end{array}$ & In build-up: 2018 & $2 / 2$ & $\begin{array}{l}\text { GSO: } 4 / 2 \\
\text { GEO: } 3 / 1\end{array}$ & 42164 & $\begin{array}{c}29 \\
0\end{array}$ \\
\hline
\end{tabular}

Table 1: Overview of global and regional navigation satellite systems

\begin{tabular}{|c|c|c|c|c|c|}
\hline GNSS constellation & $\begin{array}{c}\text { Signal } \\
\text { name }\end{array}$ & $\begin{array}{c}\text { Frequency } \\
(\mathbf{M H z})\end{array}$ & $\begin{array}{c}\text { Max beam- } \\
\text { width }\left(^{\circ}\right)\end{array}$ & $\begin{array}{c}\text { Minimum received } \\
\text { power (dBW) }\end{array}$ & $\begin{array}{c}\text { Minimum } \\
\text { radiated transmit } \\
\text { power (dBW) }\end{array}$ \\
\hline GPS & L1 C/A & 1575.42 & 23.5 & -184 & 9.1 \\
\hline Galileo & E1 B/C & 1575.42 & 20.5 & -182.5 & 10.9 \\
\hline GLONASS & L1 & 1605.375 & 20 & -179 & 14.1 \\
\hline BDS MEO & B1 & 1575.42 & 25 & -184.2 & 9 \\
\hline BDS GEO/IGSO & B1 & 1575.42 & 19 & -185.9 & 9 \\
\hline QZSS & L1 C/A & 1575.42 & 22 & -186.1 & 9.0 \\
\hline GPS & L5 & 1176.45 & 26 & -182 & 8.5 \\
\hline Galileo & E5a & 1176.45 & 23.5 & -182.5 & 8.4 \\
\hline GLONASS & L3 & 1201 & 28 & -178 & 12.6 \\
\hline BDS MEO & B2 & 1191.795 & 28 & -182.8 & 8 \\
\hline BDS GEO/IGSO & B2 & 1191.795 & 22 & -184.4 & 8.1 \\
\hline QZSS & L5 & 1176.45 & 24 & -183.4 & 9.2 \\
\hline NavIC & L5 & 1176.45 & 16 & -184.54 & 7.8 \\
\hline
\end{tabular}

Table 2: SSV signal characteristics for each GNSS service provider

\section{Assumptions for the Transmitting Antenna Pattern}

On-board the GNSS spacecraft, a simplified pattern has been modelled, assuming a constant gain for off-boresight angles below the maximum beam-width, and assuming that no signal is transmitted for higher off-boresight angles. Figure 3 illustrates the simplified GNSS antenna pattern used in the simulations. The reason behind this assumption is to generate conservative results, not considering the second lobes of the transmitted signals. 

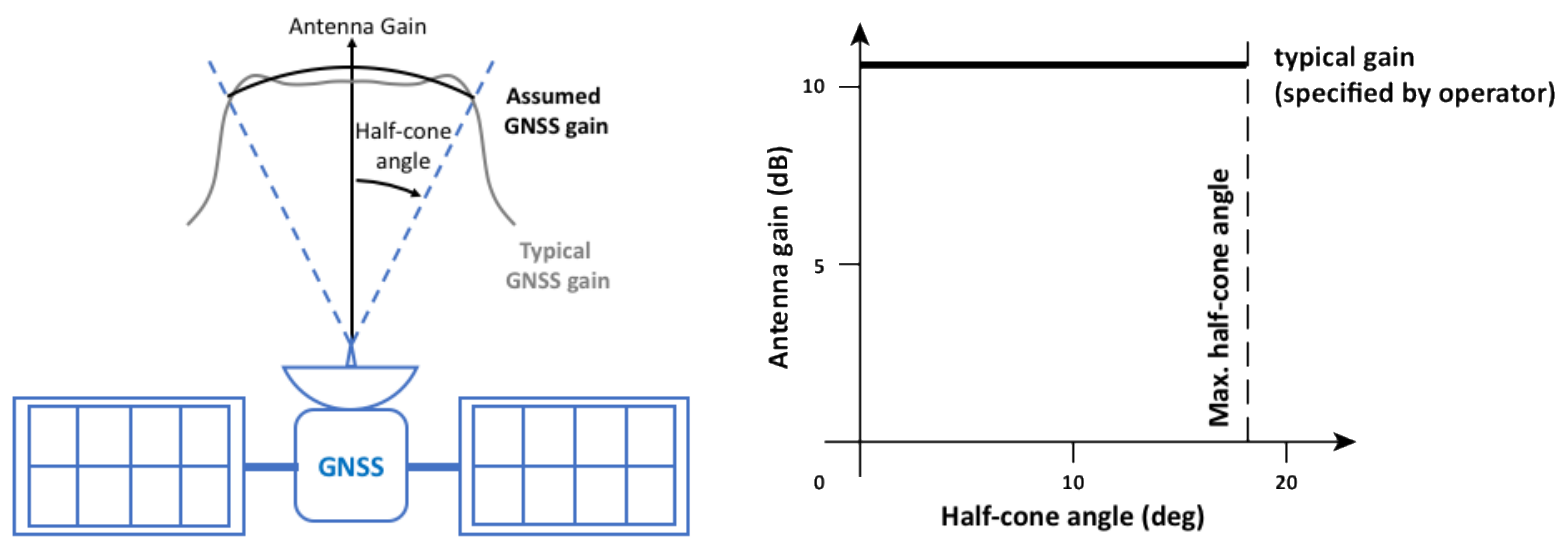

Figure 3: Simplified GNSS satellite antenna pattern, as used in the GNSS SSV simulations

\section{Assumption for Space User Antenna}

For the global analysis performed in the lower and upper SSV a zero-gain nadir-pointing antenna is assumed for all the receivers. For the other missions, a zenith-pointing patch antenna and a high-gain nadir-pointing antenna have been assumed, as shown in Figure 4 and these patterns correspond to characteristics of readily-available antennas available on the open market. All the antennas have an off-boresight cut-off angle of 90 degrees. Furthermore, for the global analysis three different acquisition thresholds of the space user receivers have been assumed: $15 \mathrm{dBHz}, 20 \mathrm{dBHz}$, and $25 \mathrm{dBHz}$. For the mission-like configurations, an acquisition threshold of the space user receiver of $20 \mathrm{dBHz}$ was assumed.

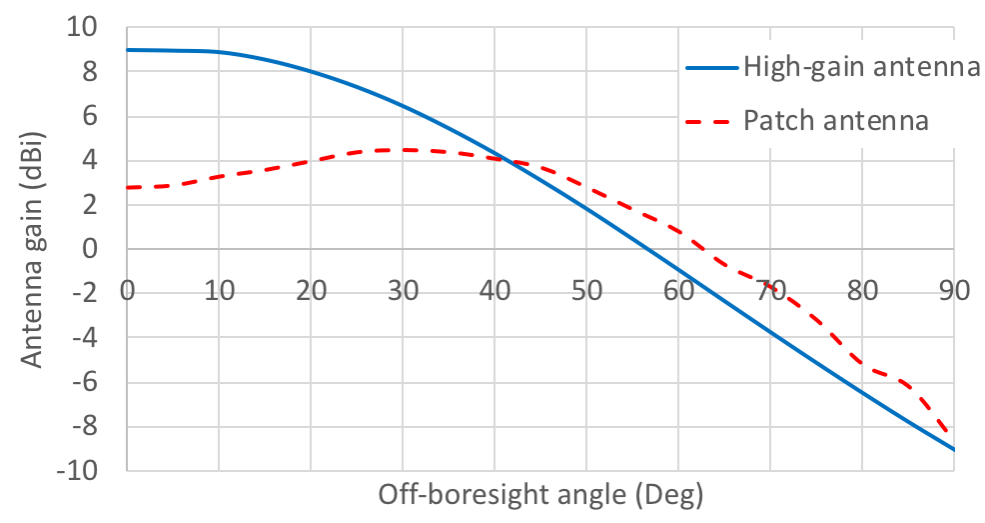

Figure 4: Simplified receiver high-gain and patch antenna patterns, as used in the GNSS SSV simulations

\section{Assumptions for Link Budget Calculations}

Figure 5 shows the approach considered in the simulations for the computation of the link budget, namely between the transmitter and receiver antennas. It is left to the reader to correct the provided statistics considering the low noise amplifiers, and other receiver specific parameters. Within this link-budget area, the parameters involved in the received C/N0 computation are: the Minimum Radiated Transmit Power (MRTP), approved by the each GNSS service provider, and reported in Table 2, the transmitter and receiver antenna patterns and the user receiver C/N0 acquisition threshold, as defined in the previous section, the free space path loss (the signal attenuation along the line of sight) computed based on the transmitter-receiver distance and the signal frequency and the system noise temperature, assumed equal to $290 \mathrm{~K}$. 


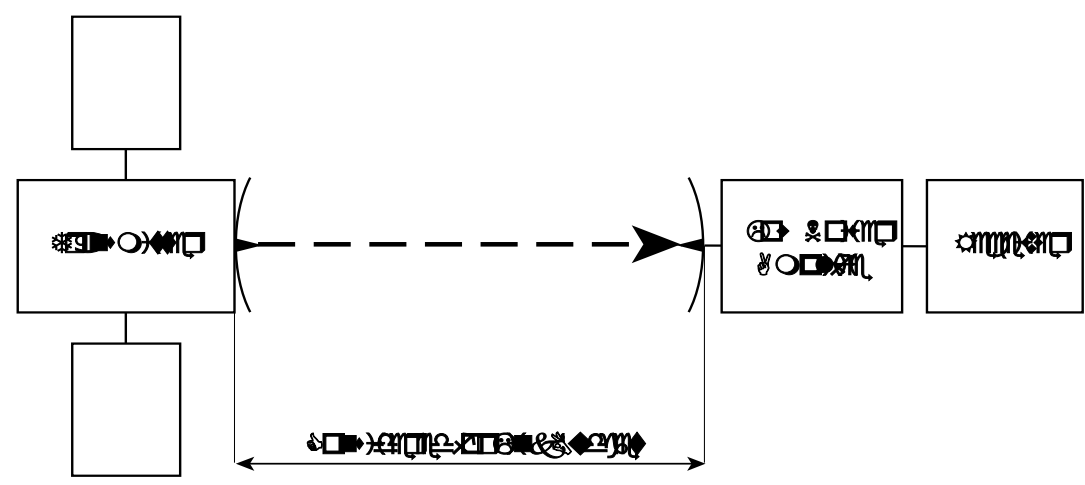

Figure 5: Link Budget calculation scenario, where the transmitter is on-board the GNSS satellite, and the Low Noise Amplifier and receiver are on-board the User spacecraft

\section{Global SSV Configuration}

The Global SSV Performance analysis performed in the lower and upper SSV has been carried out by simulating a network of user-receivers in orbit. Figure 6 shows the receiver locations using an equal-area grid of points. Each point represents a receiver's fixed ground track location on the Earth's surface from its target MEO or GEO altitude. The grid is specifically equal-area so that results computed using the points are not biased to regions containing many more points. It has roughly $4^{\circ}$ spacing near the Equator and comprises 2562 points, as shown in Figure 6.

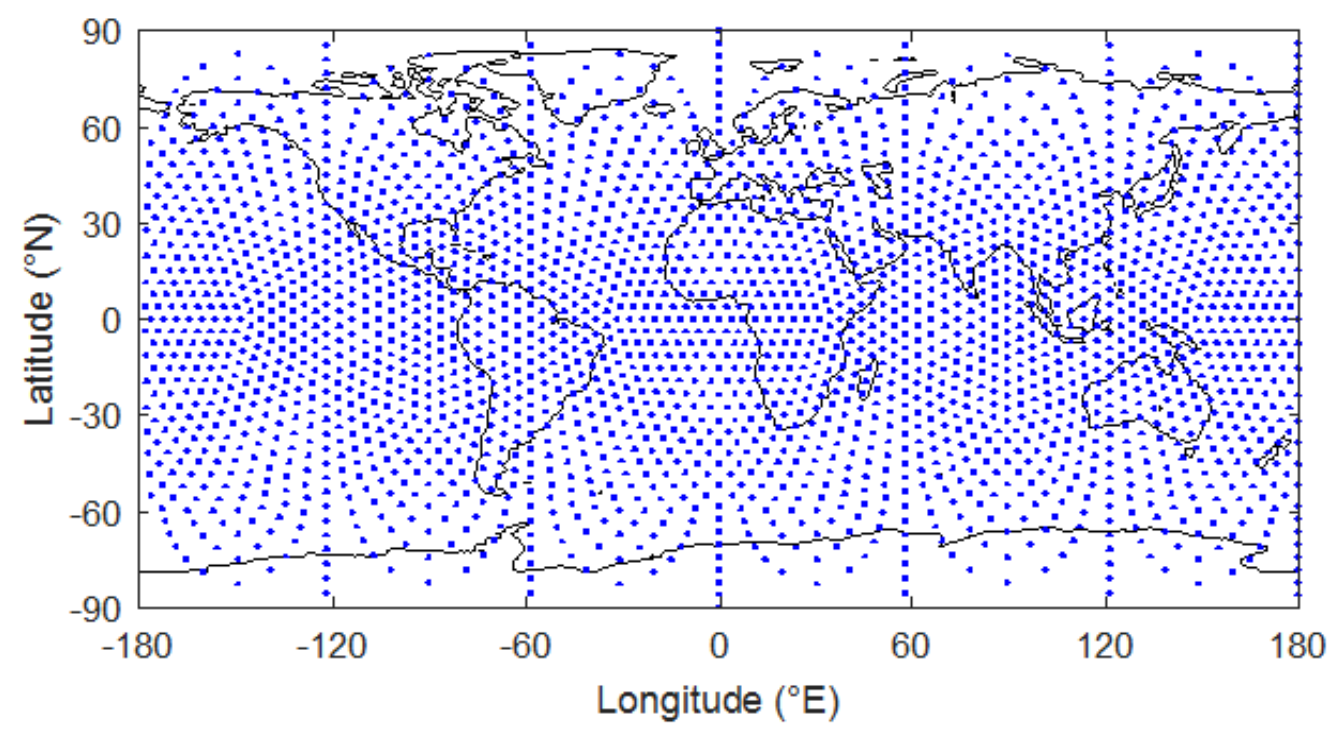

Figure 6: Space User grid location, projected over Earth's surface

\section{Mission-Specific Configuration}

The GEO mission scenario analyses multi-GNSS signal reception for six geostationary satellites with 0-degree inclination, which share the same orbital plane apart from a 60-degree separation in longitude, as shown in Figure 7. This placement of the satellites was chosen to ensure that even signals from regional GNSS satellites in (inclined) geosynchronous orbits would be visible to at least one of the GEO user receivers. The user antenna on-board the user spacecraft is a high-gain antenna that permanently points towards the nadir (center of the Earth), whose pattern is shown Figure 3. The assumed acquisition threshold of the space user receiver is $20 \mathrm{dBHz}$. 


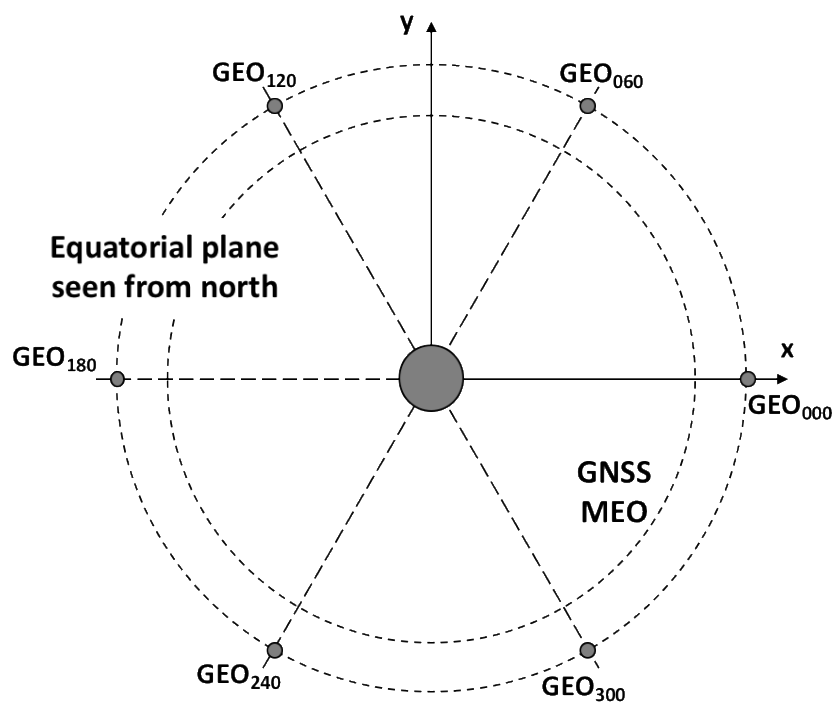

Figure 7: Schematic of the GEO mission with the 6 satellites at 60 degrees separation. The subscripts 000 to 300 correspond to the local Earth longitude.

The HEO mission scenario, shown in Figure 8, with apogee altitude of about $58600 \mathrm{~km}$ and perigee altitude of $500 \mathrm{~km}$ is used to demonstrate the GNSS visibility performance through all the GNSS SSV altitudes, both below and above the GNSS constellations. GNSS visibility conditions near the perigee are similar to those of space user receivers in LEO, with the important difference being that the spacecraft is moving very fast - around $8 \mathrm{~km} / \mathrm{s}$ to $11 \mathrm{~km} / \mathrm{s}$ - so that extreme Doppler shifts occur on the GNSS signals, and visibility times between any particular GNSS satellite and the HEO space user receiver are much shorter than for terrestrial receivers. The on-board GNSS antennas are configured in both nadir and zenith facing sides of the spacecraft. As shown in Figure 8 the nadir pointing antenna with high-gain and narrow-beam width can ensure the GNSS signal link from the opposite side of the earth, including when flying above the GNSS altitude and during the apogee period. The zenith pointing patch antenna can provide visibility during the perigee period. The antenna patterns for both type of antennas are displayed in Figure 3. The acquisition and tracking thresholds of the user receiver were both set to $20 \mathrm{~dB}-\mathrm{Hz}$ when evaluating the signal availability in the HEO simulation.

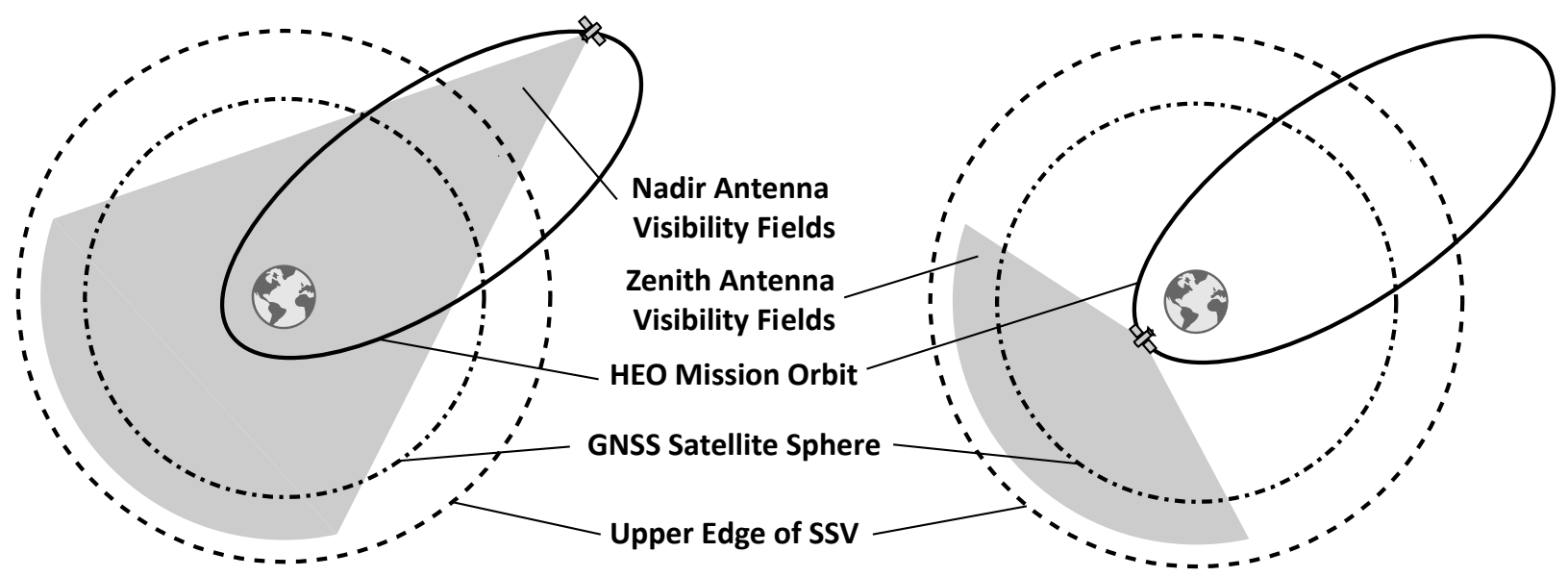

Figure 8: Schematic of the HEO mission with nadir and zenith pointing antennas 
The Lunar Mission case models a simple ballistic cislunar trajectory from low-Earth orbit to lunar orbit insertion, similar to the trajectories flown by the 1968 U.S. Apollo 8 mission and many others. This case seeks to explore the boundaries of the GNSS SSV beyond Earth orbit. The full lunar mission trajectory, as shown in Figure 9, contains four phases: 1) Earth parking orbit, 2) outbound trajectory, 3) lunar orbit, 4) return trajectory. For the purpose of this analysis only the outbound trajectory is modelled. A generic spacecraft is modelled, with two GNSS antennas: one zenith-pointing with peak gain less than $5 \mathrm{~dB}$ for reception at low altitudes, and one nadir-pointing with peak gain of approximately $10 \mathrm{~dB}$ for reception above the GNSS constellations. The results presented assume that the antenna with the greatest number of tracked satellites is used. As in the other HEO and GEO cases, the acquisition and tracking thresholds were both set to $20 \mathrm{~dB}-\mathrm{Hz}$

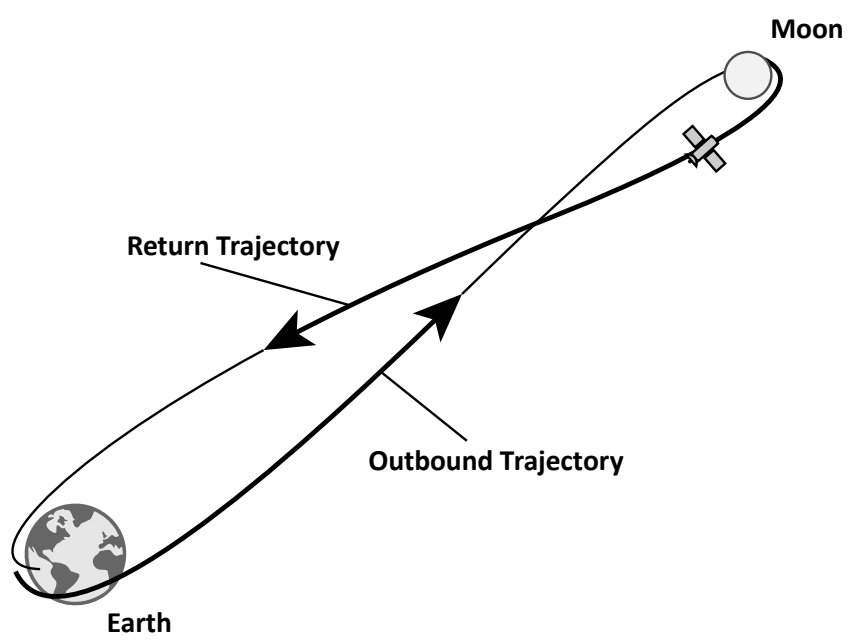

Figure 9: Lunar trajectory phases; only the outbound trajectory segment is analysed

\section{SIMULATION RESULTS AND DISCUSSIONS}

In this paragraph, the performances of the simulated scenarios are summarized and discussed, and the two most significant SSV parameters have been evaluated:

1. Signal Availability: an available signal from a GNSS satellite is one that a space user with adequate equipment is able to detect with sufficient strength to form a usable measurement, i.e. above the carrier power to noise power spectral density (C/N0) threshold value required to acquire and track the signal, and with unobstructed line of sight. The signal availability is measured as a statistic over both location and time and the percentage of GNSS signals available for use by a space user are reported. It is calculated both as the availability of a single signal in view, and as the availability of four signals in view, to capture the various requirements of space users. In both cases, in order to declare a signal available, it needs to be both:

a. received at a signal power level higher than the minimum specified for SSV users,

b. and observed with a user range error smaller than the maximum user range error specified for SSV users.

2. Maximum Outage Duration: is defined as the maximum duration when a space user at a particular orbit will not obtain availability for at least one single signal or at least four signals simultaneously, depending on the exact metric being calculated. The definition of maximum outage duration is closely linked to the definition of signal availability.

\section{Global SSV Performance in the Upper SSV}

Figure 10 shows an example of simulated signal availability for the $20 \mathrm{~dB}-\mathrm{Hz}$ C/N0 threshold case for a 24 hours-arc. Note how each single GNSS constellation, individually, contributes with an average of about 2 satellites visible per epoch, up to a maximum of 6 visible satellites in some particular occasions. On the other hand, if all the constellations are considered together, the number of visible GNSS satellites is never below 4, spanning to a maximum of 14 satellites. Table 3 summarizes the results for the upper $\mathrm{SSV}$, in terms of at least 1 signal availability, at least 4 signals availability and maximum outage duration. 


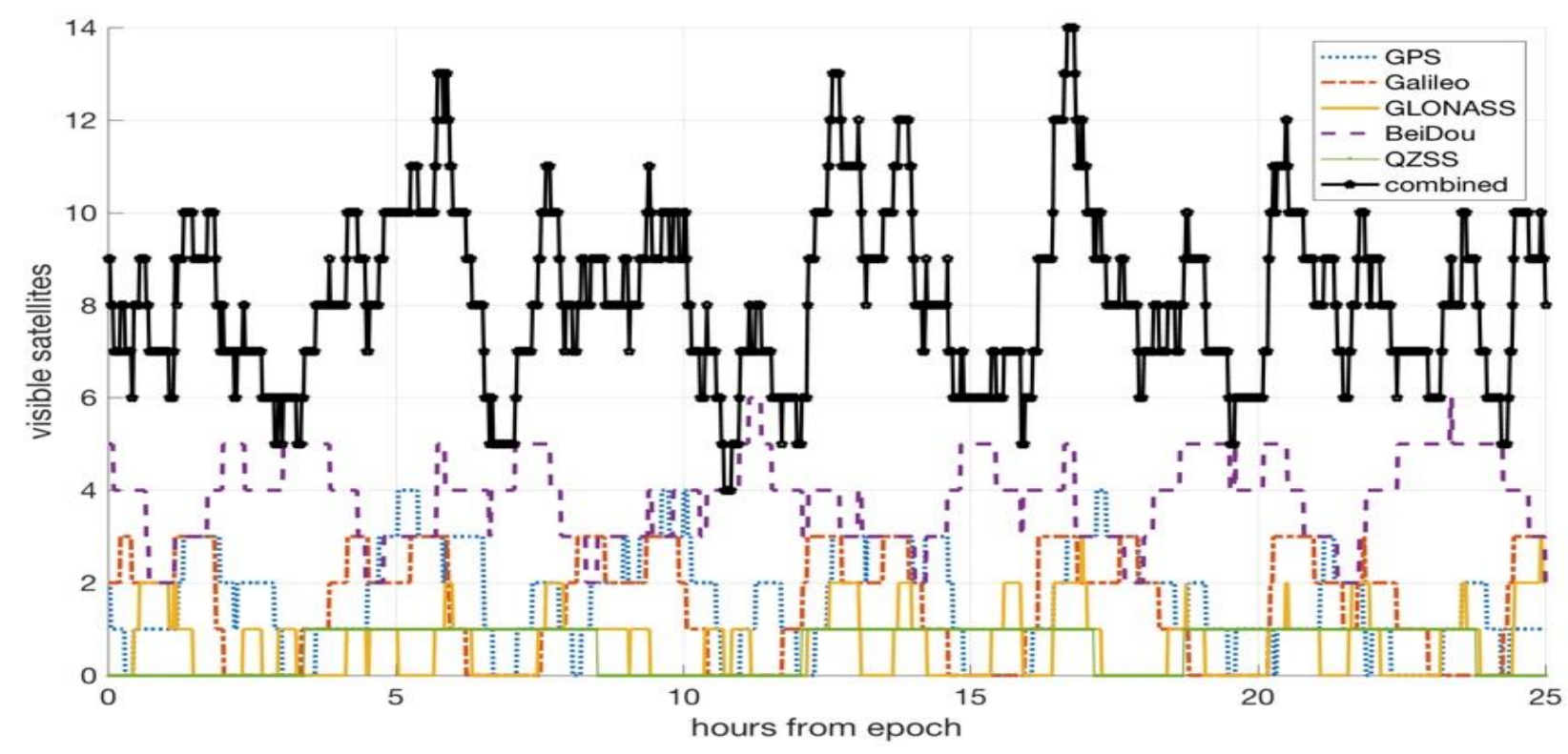

Figure 10: Estimated number of satellites visible, by individual constellation and combined, for sample GEO user with $20 \mathrm{~dB}-\mathrm{Hz}$ C/NO threshold.

General observations concerning the results shown in Table 3 indicate the following:

- One-signal availability significantly exceeds four-signal availability, underscoring the benefit of employing an on-board navigation filter, which can process individual measurements at a time, for missions in the SSV.

- When the constellations are used together and two frequencies are tracked, the one-signal availability is $100 \%$.

- When the constellations are used together and two frequencies are tracked, the at least 4-signal availability is between 89.9 and $99.9 \%$, depending whether the signals on both frequencies needs to be available at the same time.

- The abundance of signals available in an interoperable multi-GNSS SSV greatly reduces constraints imposed by navigation at high altitudes.

Table 3: Global performance estimates of availability and MOD for global and regional constellations and all constellations together. Results for nadir pointing 0 dBi gain antenna in the Upper SSV, with $20 \mathrm{dBHz}$ minimum received $\mathrm{C} / \mathrm{NO}$ threshold.

\begin{tabular}{|c|c|c|c|c|c|}
\hline \multirow[b]{2}{*}{ Band } & \multirow[b]{2}{*}{ Constellation } & \multicolumn{2}{|c|}{ Signal Availability in \% } & \multicolumn{2}{|c|}{ Max Outage Duration in min } \\
\hline & & $\begin{array}{c}\text { At least } 1 \\
\text { Signal }\end{array}$ & $\begin{array}{c}4 \text { or more } \\
\text { Signals }\end{array}$ & $\begin{array}{c}\text { At least } 1 \\
\text { Signal }\end{array}$ & $\begin{array}{c}4 \text { or more } \\
\text { Signals }\end{array}$ \\
\hline \multirow{3}{*}{ L1/E1/B1 } & Global Systems & $79-94$ & $0.6-7$ & $48-111$ & $*$ \\
\hline & QZSS & 0 & 0 & $*$ & $*$ \\
\hline & Combined & 99.9 & 89.9 & 33 & 117 \\
\hline \multirow{3}{*}{ L5/L3/E5a/B2 } & Global Systems & $93-99.9$ & $4-60$ & $7-77$ & $1180^{*} *$ \\
\hline & Regional Systems & $1-30$ & $0-1.5$ & $*$ & $*$ \\
\hline & Combined & 100 & 99.9 & $\mathbf{0}$ & 15 \\
\hline
\end{tabular}

\footnotetext{
*No signal observed for the worst-case grid location for maximum simulation. This means that there is at least one receiver that will never receive more than 3 signals.
} 
Global performance estimates of availability and MOD for the lower SSV (represented by a user sphere at $8000 \mathrm{~km}$ altitude) are shown in Table 4. Performance in the lower SSV is estimated to be significantly better than that in the upper SSV, due to the improved geometric availability at the lower altitude. Single-satellite availability is nearly $100 \%$ for all individual systems and combined-constellation availability is $100 \%$ in all cases. For the lower SSV, the C/N0 is typically higher than the assumed $20 \mathrm{~dB}$ $\mathrm{Hz}$ minimum tracking threshold. This means that the simulations conducted here (geometry-based only) are considered to be realistic because the assumed GNSS receiver tracking/acquisition threshold of $20 \mathrm{dBHz}$ is much lower than the signal strength available at this altitude. This statement is based on the analysis of real GNSS data at the respective orbital altitude.

Table 4: Global performance estimates of availability and MOD for global and regional constellations and all constellations together. Results for 0 dBi omni pointing antenna (nadir and zenith) in the Lower SSV.

\begin{tabular}{|c|c|c|c|c|c|}
\hline \multirow{3}{*}{ Band } & \multirow{3}{*}{ Constellation } & \multicolumn{2}{|c|}{ Signal Availability in \% } & \multicolumn{2}{c|}{ Max Outage Duration in min } \\
\cline { 2 - 6 } & $\begin{array}{c}\text { At least 1 } \\
\text { Signal }\end{array}$ & $\begin{array}{c}\mathbf{4} \text { or more } \\
\text { Signals }\end{array}$ & $\begin{array}{c}\text { At least 1 } \\
\text { Signal }\end{array}$ & $\begin{array}{c}\text { 4 or more } \\
\text { Signals }\end{array}$ \\
\hline \multirow{3}{*}{ L1/E1/B1 } & Global Systems & $99.9-100$ & $95-100$ & $0-11$ & $0-60$ \\
\cline { 2 - 6 } & QZSS & 99.6 & 79.4 & 197 & $*$ \\
\cline { 2 - 6 } & Combined & $\mathbf{1 0 0}$ & $\mathbf{1 0 0}$ & $\mathbf{0}$ & $\mathbf{0}$ \\
\hline \multirow{3}{*}{ L5/L3/E5a/B2 } & Global Systems & 100 & $99.9-100$ & 0 & $0-16$ \\
\cline { 2 - 7 } & Regional Systems & $98-99.6$ & $51-79$ & $197-348$ & $*$ \\
\cline { 2 - 7 } & Combined & $\mathbf{1 0 0}$ & $\mathbf{1 0 0}$ & $\mathbf{0}$ & $\mathbf{0}$ \\
\hline
\end{tabular}

* No signal observed for the worst-case grid location for maximum simulation. This means that there is at least one receiver that will never receive more than 3 signals.

\section{Mission-Specific Performance - GEO}

The MEO GNSS satellites have orbital periods in the order of 12 - 14 hours, or about half that of the GEO. This means that the GEO and MEO orbits are almost in phase with each other, in such a way that the visibility patterns at the GEO receiver repeat almost exactly with periods of one day. This means that the visibility patterns in terms of number of visible MEO signals are very similar to all six GEO receivers, and the visibility pattern can be observed in Figure 11 (figure on top).

The situation is different for the inclined geosynchronous GNSS satellites of the NavIC, QZSS and BDS constellations. The GEO and IGSO longitudes are frozen relative to each other. At most GEO longitudes, the GNSS satellites in IGSO orbits are never visible, either because the GEO is located outside the half-cone angle of the transmitting satellite, or because the signal is blocked by the Earth. This means that reception of the IGSO GNSS signals is an exception rather than the rule. However, those GEO receivers that do see signals from these transmitters will see them continuously, or at very regular patterns (see NavIC B2/E5A/L5 signal), as can be seen in Figure 11 (bottom figure).

For GEO longitudes where no BDS, QZSS or NavIC geosynchronous satellites are visible (from 0 to 180 degrees), there are typically not more than three L1 signals available from any individual GNSS constellation. Combined, there are almost always four or more signals, and often up to ten signals.

For L5, the individual constellations are slightly better than for L1, and often provide four signals. The combined constellations provide almost always six or more signals, up to fifteen and more. Note in particular the presence of BDS signals at GEO 300, which brings the combined visibility above fifteen satellites through most of the simulation period. 


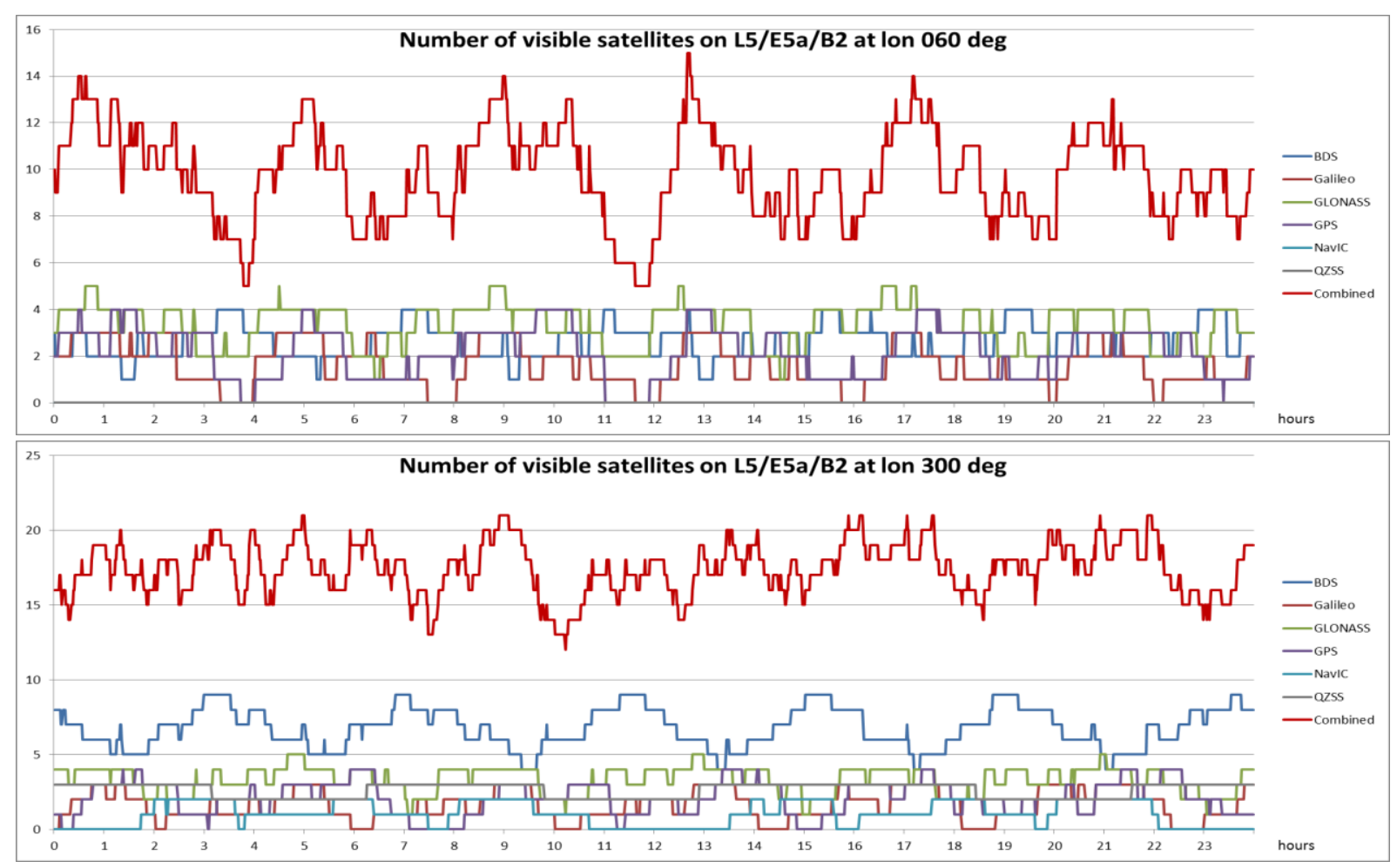

Figure 11: example of L5/E5a/B2 visibility for GEO at 60 degrees and 300 degrees

\section{Mission-Specific Performance - HEO}

Figure 12 shows the GNSS signal availability of all GNSS constellations and L5/L3/E5a/B2 signal for the HEO nadir and zenith pointing antennas over the time of $1.5 \mathrm{HEO}$ orbital periods. Note that when the spacecraft is below the GNSS constellation altitude, the visibility can be significantly improved by combining the signals from both Nadir and Zenith antennas at the same time. However, within this simulation only the strongest signal from either is employed at a given time. Around apogee, only the nadir pointing antenna provides signal availability.

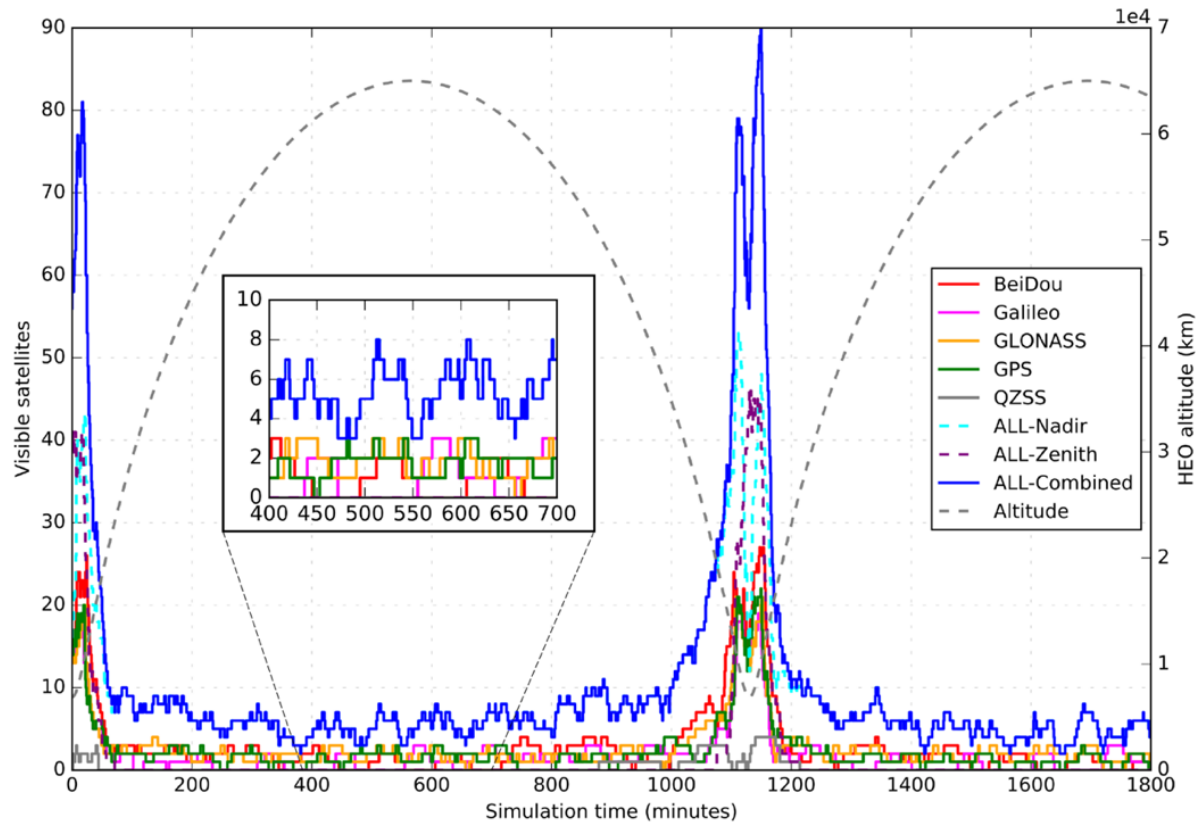

Figure 12: Visible GNSS satellites over 1.5 orbital periods of HEO (L5/L3/E5a/B2) 
The simulated results for the signal availability and maximum outage duration (MOD) of the HEO mission are shown in Table 5. For both L1/E1/B1 and L5/L3/E5/B2 the one-signal availability can reach 100\% with all constellations combined. In case of L1, four-signal availability is below $20 \%$ and the maximum outage duration is around 1000 minutes, which is close to the HEO orbital period of 1130 minutes, for an individual constellation. The performance is significantly improved by receiving signals from all constellations combined for both signals L1 and L5, where the signal L5 performs better.

Table 5: HEO mission simulated performance result

\begin{tabular}{|c|c|c|c|c|c|}
\hline \multicolumn{6}{|c|}{ Nadir -and Zenith Antennas Combined } \\
\hline \multirow{3}{*}{ Band } & \multirow{3}{*}{ Constellation } & Signal Availability in \% & \multicolumn{2}{c|}{ Max Outage Duration in min } \\
\cline { 3 - 6 } & & $\begin{array}{c}\text { At least 1 } \\
\text { Signal }\end{array}$ & $\begin{array}{c}\mathbf{4} \text { or more } \\
\text { Signals }\end{array}$ & $\begin{array}{c}\text { At least 1 } \\
\text { Signal }\end{array}$ & $\begin{array}{c}\text { 4 or more } \\
\text { Signals }\end{array}$ \\
\hline \multirow{3}{*}{ L1/E1/B1 } & Global Systems & $74-98$ & $9-16$ & $12-85$ & $986-1036$ \\
& QZSS & 27.5 & 2.5 & 1031 & 2175 \\
\cline { 2 - 6 } & Combined & $\mathbf{1 0 0}$ & $\mathbf{9 4 . 5}$ & $\mathbf{0}$ & $\mathbf{4 7}$ \\
\hline \multirow{3}{*}{ L5/L3/E5a/B2 } & Global Systems & $87-100$ & $12-56$ & $0-63$ & $133-980$ \\
& Regional Systems & $32-35$ & 6 & $989-1021$ & 1091 \\
\cline { 2 - 6 } & Combined & $\mathbf{1 0 0}$ & $\mathbf{1 0 0}$ & $\mathbf{0}$ & $\mathbf{0}$ \\
\hline
\end{tabular}

\section{Mission-Specific Performance - Lunar}

Table 6 contains the simulated performance results for this mission. In the case of both L1 and L5 bands, the availability of 4 simultaneous signals is nearly zero for any individual constellation, though combined there is coverage to approximately 30 Earth Radii (RE) (approximately half the distance to the Moon), near 10-15\% in average. Single-satellite availability reaches a $36 \%$ average for the combined case at L5, though as shown in Figure 13, this availability primarily occurs at low altitudes. The benefit of the combined case is best seen above $10 \mathrm{RE}$, where the combined case has signal availability significantly higher than any individual constellation, and often nearly double. Notably, combining constellations does not increase the altitude at which such signals are available; rather, it increases the number of signals available at a given altitude.

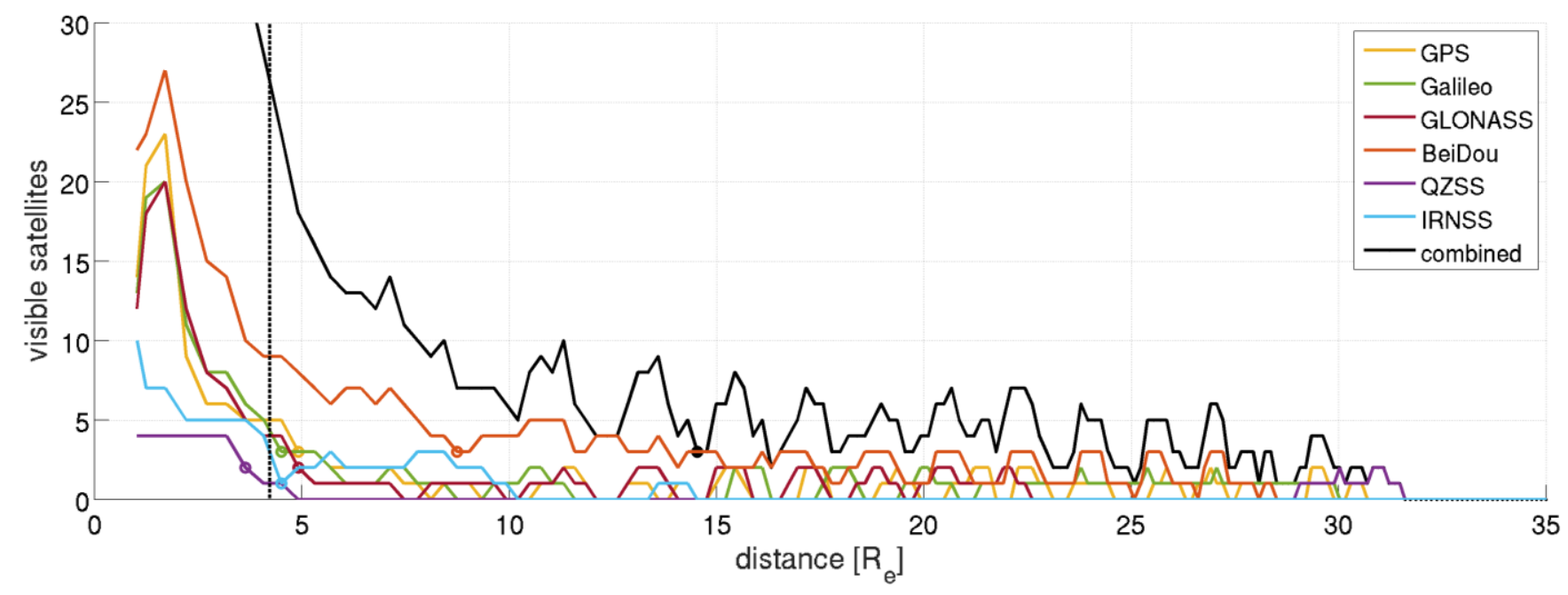

Figure 13: Signal visibility by trajectory altitude, to the limit of available signals at 30 RE (approx. 50\% of lunar distance).

Table 6: Lunar mission simulated performance results 


\begin{tabular}{|c|c|c|c|}
\hline \multirow{2}{*}{ Band } & \multirow{2}{*}{ Constellation } & \multicolumn{2}{|c|}{ Signal Availability in \% } \\
\cline { 3 - 4 } & & At least 1 signal & 4 or more signals \\
\hline \multirow{2}{*}{ L1/E1/B1 } & Global Systems & $8-14$ & $1-3$ \\
& QZSS & 1 & 0 \\
\cline { 2 - 4 } & Combined & $\mathbf{2 1}$ & $\mathbf{9}$ \\
\hline \multirow{2}{*}{ L5/L3/E5a/B2 } & Global Systems & $12-33$ & $1-5$ \\
& Regional Systems & 4 & $0-1$ \\
\cline { 2 - 4 } & Combined & $\mathbf{3 6}$ & $\mathbf{1 6}$ \\
\hline
\end{tabular}

Figure 14 shows the simulated C/N0 received by the example spacecraft for each individual constellation. The figure shows the reason for the visibility drop-off near $30 \mathrm{RE}$ (as depict in Figure 13), because the C/N0 of signals at the receive antenna drops below the $20 \mathrm{~dB}-\mathrm{Hz}$ minimum threshold beyond that point. Figure 14 provides a very important message: in case a more advanced GNSS equipment (sensitive GNSS receiver with the capability to track weak GNSS signals in combination with the use of a high-gain antenna) would be available, so that the acquisition/tracking threshold of the GNSS receiver is in the order of $15 \mathrm{~dB}-\mathrm{Hz}$, than signal availability would be achievable for the entire trajectory to lunar distance!

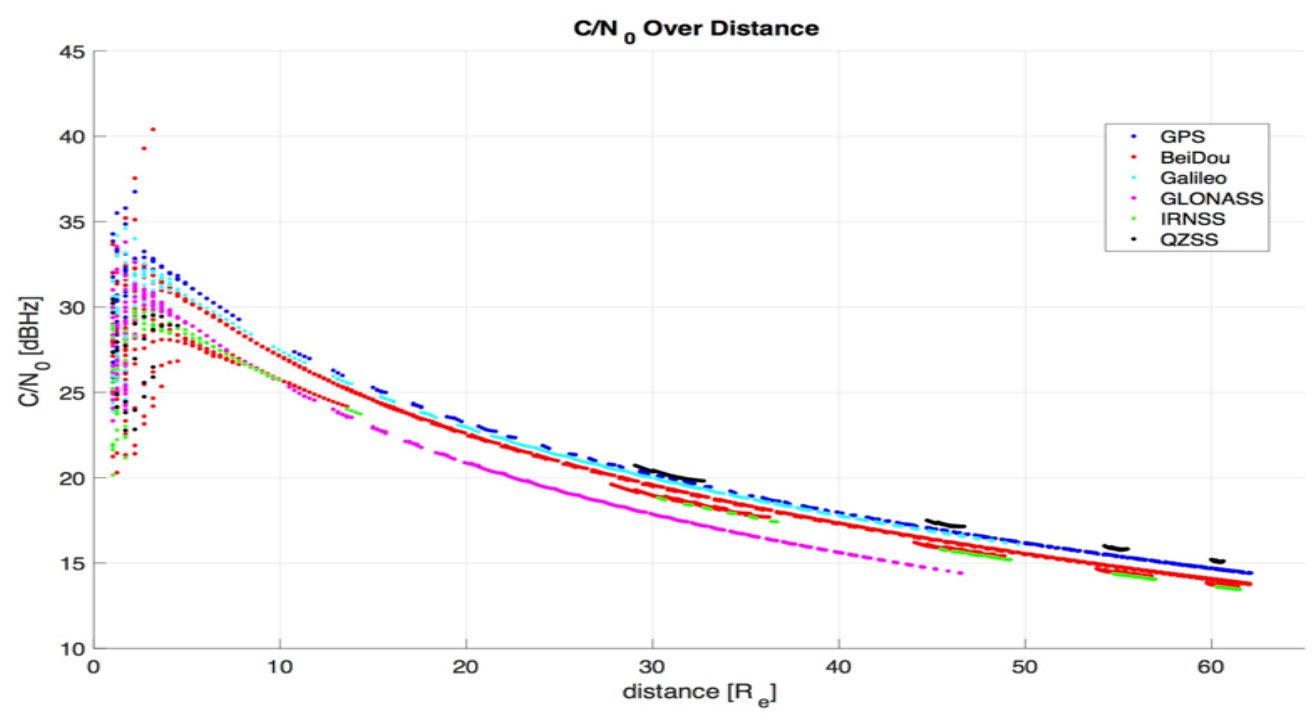

Figure 14: Simulated C/NO for lunar trajectory

\section{CONCLUSIONS}

Several scenarios related to the interoperable GNSS SSV were simulated and analysed. The range of the simulations covered Global SSV performance and also three realistic mission scenarios, namely GEO, HEO and a Lunar Transit trajectory. The focus of the simulations was the analysis of Signal Availability and Maximum Outage Time, as tow of the most important parameters for the GNSS SSV. For this reason, a set of fundamental assumptions have been made, addressing constellations configuration, GNSS and end user antenna pattern, general GNSS receiver equipment characteristics, location of antennas on user spacecraft and attitude of user spacecraft, allowing a realistic link budget calculation. In this context, about 49 million signals were simulated, processed, analysed and compared. The results from ESA, NASA, CAST and ISRO showed a consistency within $0.015 \mathrm{dBHz}$ for the link budget calculations. The outcomes of the simulations showed very clearly that there are many benefits resulting from an interoperable GNSS SSV, including increased signal availability for high-altitude users compared to that provided by any individual constellation alone, increased geometric diversity and thus accuracy in the final navigation solution, increased responsiveness, increased level of autonomy due to reduced signal outages also and increased resiliency due to the diversity of signals and constellations used. These benefits are truly enabling for classes of emerging advanced users, including ultra-stable remote sensing from geostationary orbit (GEO), agile and responsive formation flying, and more efficient utilization of valuable slots in the GEO belt. 
There are significant signal availability improvements compared to any individual constellation when all GNSS constellations are employed. Within the high-altitude interoperable GNSS SSV, single-signal availability reaches nearly $100 \%$ for the L1/E1/B1- band, and four-signal availability jumps from a maximum of $7 \%$ for any individual constellation to about $90 \%$ with usage of signals from all constellations. The maximum signal outage duration for 1 signal availability is hereby only 33 minutes for L1/E1/B1 band and for the case of 4 signals or more in the same band the maximum signal outage duration is 117 minutes.

The outcomes of the simulations considering the link-budget, demonstrating very clearly the enormous potential that the combined use of an interoperable GNSS Space Service Volume can offer to high-altitude missions, which are flying outside the geostationary belt, such as high-elliptical orbits and also missions to the Moon. In this context, it is important to note that for the simulations the assumptions about the GNSS equipment are considered as conservative, because the characteristics of existing commercial of the shelf receivers and antennas have been used. In addition, only the signals in the main lobe have been taken into account and no side lobes signals have been considered.

This means that the existing true potential of the interoperable GNSS SSV is in reality much bigger, as the simulations results of the three scenarios indicate. The reasons for this statement are: the usage of advanced GNSS receiver technology with the capability of tracking weak signals, the usage of specific developed high gain antennas and also the fact that signals from the side lobe can be tracked. Hence, the combined signal availability (main lobe + side lobe) is definitely larger than the signal availability of only the main lobe. Taking the case of the Moon mission into account, it has been shown that by using advanced GNSS equipment (receiver and antenna) it would be possible to track GNSS satellites all the way to the Moon and therefore opening the field to ground-breaking opportunities.

The analyses presented in this paper clearly show the enormous potential of the GNSS SSV, the wide range of significant benefits and therefore the importance of an interoperable GNSS Space Service Volume for space users in general and in particular for high-altitude space users.

\section{ACKNOWLEDGMENTS}

Sincere thanks to the co-chairs of ICG Working Group-B (WG-B) on "Enhancement of GNSS Performance, New Services and Capabilities" of the United Nations International Committee on GNSS (ICG) and all the Working Group members, who participated in the GNSS SSV activities. Special thanks to the following contributing agencies (in alphabetic order): CAO, CAST, ESA, FBauer Aerospace Consulting Services, George Washington University, ISAC/ISRO, ISS-Reshetnev Company, JAXA, NASA GRC, SCaN, NEC, Overlook Systems Technologies Inc, PNT Center, TSNIIMASH, State Space Corporation Roscosmos, SAC/ISRO and SJTU.

\section{REFERENCES}

1. SSV Booklet: ICG Working Group-B (WG-B), The Interoperable GNSS Space Service Volume, Booklet, 2018

2. J. Parker, F. Bauer, B. Ashman, J. Miller, W. Enderle, D. Blonski, Development of an Interoperable, Multi-GNSS Space Service Volume, ION GNSS+ 2018 